\title{
Implementación de entrenamiento en simulación para la formación de estudiantes de medicina de pregrado: retención de conocimientos en reanimación cardiopulmonar
}

\author{
Implementing simulation training in undergraduate medical training: \\ knowledge retention on cardiopulmonary resuscitation \\ Carolina Felipe Soares-Brandão,* Dario Cecilio-Fernandes, ${ }^{\ddagger}$ Heimar de Fatima Marin ${ }^{\S}$
}

Palabras clave: Educación médica, formación médica universitaria, entrenamiento de simulación, metodologías de enseñanza y aprendizaje.

Keywords:

Medical education, undergraduate medical training, simulation training, teaching-learning methodologies.

* Universidade Federal de São Paulo (UNIFESP) y Universidade Cidade São Paulo (UNICID), Brasil.

‡ Faculdade de Ciências Médicas, Universidade Estadual de Campinas (UNICAMP), Brasil. $\S$ Programa de Pregrado en Informática de

Tratamiento y Salud, Universidade Federal de São Paulo-UNIFESP, São Paulo, Brasil.

\section{RESUMEN}

Introducción: El entrenamiento con simulación ha demostrado lograr la integración del conocimiento y las habilidades de los alumnos. Si bien, mucho interés se ha puesto en el entrenamiento con simulación en alumnos de postgrado, poco se ha estudiado sobre su uso en estudiantes de medicina. Por consiguiente, este estudio tiene por objetivo evaluar la adquisición y retención de conocimiento de los estudiantes de pregrado después de un entrenamiento de simulación en emergencia cardiovascular. También investigamos la relación entre el conocimiento de los estudiantes y el número de pacientes reales con emergencias cardiovasculares tratados. Material y métodos: Estudio observacional, longitudinal y cuantitativo realizado en la Universidad Cidade São Paulo (UNICID). Durante su pasantía, los estudiantes asistieron al curso de capacitación de simulación clínica, cuatro veces durante un periodo de dos años; con el objetivo de aplicar sus conocimientos y habilidades sobre emergencia cardiovascular avanzada. Después de cada sesión, los alumnos contestaron una prueba de conocimientos. También registramos la participación de los estudiantes en la capacitación simulada y si habían tratado algún caso de la vida real. Para analizar las puntuaciones de los estudiantes a lo largo del tiempo, se utilizó el análisis de varianza de medidas repetidas (ANOVA). Para comparar a los estudiantes que tuvieron contacto con pacientes clínicos vivos con aquellos que no lo hicieron, utilizamos la prueba t. Resultados: Hubo un aumento en el conocimiento de los estudiantes durante este periodo. No hubo diferencias en los puntajes de los estudiantes que tuvieran contacto con casos cardiovasculares en ambiente real respecto a los que no lo tuvieron. Conclusión: Aparentemente el entrenamiento en simulación favorece la adquisición de conocimientos y su retención en los estudiantes, proporcionando una estrategia eficiente y realística de entrenamiento en escuelas de formación médica. De un modo bastante interesante, no hubo diferencia entre las puntuaciones de estudiantes que atendieran pacientes con condición similar en la vida real, con aquellos que no tuvieran contacto con pacientes reales. Una futura investigación debería indagar el motivo por el cual no hubo diferencia entre ambos grupos de estudiantes.

\section{ABSTRACT}

Introduction: Simulation training has been shown to support the integration of students' knowledge and skills. Although much is known about simulation training in residency programs, little is known about the use of simulation training in undergraduate medical students. Therefore, we investigated students'knowledge acquisition and retention after a simulation training in cardiovascular emergency. We also investigated the relation between students' knowledge and the number of cardiovascular treatments on live clinical patients. Material and methods: This observational, longitudinal and quantitative study was conducted at Universidade Cidade São Paulo (UNICID). During their clerkship, students attended the course four times during two years to a clinical simulation training; so that they could apply their knowledge and skills about advanced cardiovascular emergency. After each encounter, students answered a knowledge test. We also recorded student participation in the simulated training and whether they have treated any real life cases. To analyze students' scores over time, repeated measures analysis of variance (ANOVA) was used. To compare students who had contact with live clinical patients with those who not, we used t-test. Results: There was an increase in students' knowledge over time. There was no difference on students' scores between students who had contact with live clinical patients with those who only had contact with clinical simulation. Conclusion: It seems that simulation training support students' knowledge acquisition and retention, providing an efficient and realistic training strategy in undergraduate medical schools. Interestingly, there was no difference between scores of students who had attended live clinical patient with those who had not have contact with clinical simulations. Further research should investigate the reason that there was no difference between both groups of students. 


\section{INTRODUCCIÓN}

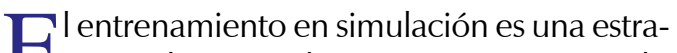
$\amalg$ tegia educacional que propone una condición médica o evento, que es proyectada para imitar la práctica real. En esta circunstancia, el entrenamiento en simulación promueve el aprendizaje a través del uso del conocimiento y habilidades de los estudiantes, ya sea en aspectos teóricos, habilidades técnicas o habilidades no técnicas. El énfasis en el entrenamiento en simulación recae en la integración de conocimiento y habilidades de los estudiantes, los cuales intensifican su pensamiento crítico y reflexivo. ${ }^{1}$ El entrenamiento en simulación también estimula el aprendizaje activo, exige la participación activa de los participantes, y motiva la práctica personal y colaborativa. ${ }^{2}$ Asimismo, el entrenamiento en simulación puede promover la profundización del conocimiento y aumentar la retención de conocimiento y habilidades a largo plazo. ${ }^{3}$

El entrenamiento en simulación se usa como una metodología de enseñanza y no sólo como una forma de exponer de manera artificial a los estudiantes a situaciones clínicas reales. ${ }^{1,4}$ En otras palabras, la simulación permite a los estudiantes experimentar eventos clínicos que de otra forma quizá no podrían presenciar, contribuyendo así a desarrollar y poner a prueba sus habilidades clínicas en esas situaciones.

El uso de entrenamiento en simulación ha demostrado mejorar las habilidades quirúrgicas, la capacidad de manejar escenarios sobre eventos cardiovasculares y la adquisición y mantenimiento de conocimientos relacionados, al compararse con conferencias y clases tradicionales. ${ }^{5}$ Por otra parte, el entrenamiento en simulación ayuda a abordar el problema de déficit de entrenamiento, información no actualizada y competencia médica. ${ }^{5}$ Además, los estudiantes que tuvieron contacto previo con entrenamiento en simulación también adquirieron habilidades fundamentales en comunicación, pues ese es un aprendizaje más realístico y significativo. ${ }^{6-8}$

Aunque la investigación haya demostrado que el entrenamiento en simulación perfecciona el conocimiento y retención de habilidades de los estudiantes, ${ }^{9}$ es muy poco conocido el uso de entrenamiento de simulación para estudian- tes de medicina no graduados. ${ }^{9,10}$ Un estudio demostró que el entrenamiento que brinda la Facultad de Medicina en el tratamiento de pacientes graves es insuficiente, y puede incluso aumentar el riesgo de error en estos pacientes. ${ }^{11}$ De este modo, incluir el entrenamiento en simulación para los estudiantes antes de iniciar su entrenamiento clínico les ayudará a enfrentar la realidad clínica, así como a dar mayor seguridad al paciente incrementando el conocimiento de los estudiantes sobre estas emergencias. En este estudio investigamos las repercusiones del entrenamiento en simulación sobre la adquisición y retención del conocimiento en emergencias cardiovasculares avanzadas.

\section{MATERIAL Y MÉTODOS}

Se incluyeron 45 estudiantes de medicina matriculados en su quinto y sexto año de la carrera de medicina. Dichos participantes fueron una muestra de conveniencia matriculados en el curso de medicina.

Este estudio observacional, longitudinal y cuantitativo ha sido llevado a cabo en la Universidade Cidade São Paulo (UNICID). La recopilación de datos ha sido realizada de febrero de 2012 hasta noviembre de 2013. Todos los estudiantes aceptaron participar en el estudio y firmaron el formulario de consentimiento informado. Acompañamos a los estudiantes durante todo su periodo de educación para determinar con qué frecuencia encontraban emergencias cardiovasculares avanzadas en el ambiente real (hospital).

Los estudiantes asistieron cuatro veces durante dos años para un entrenamiento en simulación clínica; con el objetivo de aplicar su conocimiento y habilidades sobre emergencias cardiovasculares avanzadas. Los estudiantes fueron asignados de manera aleatoria a cinco grupos en el curso de emergencias clínicas. Todos los grupos recibieron el mismo entrenamiento educacional (incluido el profesor), en el cual hubo sólo evaluación formativa. Durante el entrenamiento, de tres a cuatro voluntarios participaron en los escenarios, mientras que los estudiantes restantes observaban la situación. La participación en el curso era obligatoria (pero no en la investigación) y la ausencia de los estudiantes era reflejada en su calificación 
final; así todos los estudiantes participaron en el entrenamiento.

El entrenamiento en simulación se llevó a cabo en una sala de simulación con equipos completos de emergencias. También, usamos un simulador de alta fidelidad con recursos audiovisuales. La sesión de simulación fue dividida en una sesión de instrucciones previa, de cinco minutos de duración, en la cual se explicaron los objetivos e instrucciones a los estudiantes. Luego, asistieron a un escenario simulado de 15 minutos de duración aproximada sobre una emergencia cardiovascular avanzada. El tópico específico fue fibrilación ventricular secundaria a tromboembolia pulmonar. Aunque la información respecto al nombre, edad, anamnesis del paciente eran diferentes en cada escenario, la hemodinámica y conducta esperada fueron las mismas para todos los escenarios. Después del escenario simulado, se llevó a cabo un debriefing de 40 minutos para discutir el desempeño del grupo, el cual fue guiado por profesores experimentados en escenarios simulados.

\section{Instrumentos}

Los estudiantes fueron evaluados mediante un examen de conocimientos dos veces al año. El examen de conocimientos siguió las directrices internacionales para emergencias cardiovasculares avanzadas y fue validado por nuestro equipo de profesores además de otros tres cardiólogos de diferentes instituciones. El examen de conocimientos constó de 10 preguntas de opción múltiple sobre emergencias cardiovasculares avanzadas; cada ítem tenía cuatro respuestas alternativas y sólo una era

Tabla 1: Número de estudiantes, media, desviación típica, mínimo y máximo de notas de los estudiantes para los semestres 9, 10,11 y 12 .

\begin{tabular}{lccccc} 
& & & Desviación & & \\
& $\mathrm{n}$ & Media & típica & Mínimo & Máximo \\
\hline Semestre 9 & 33 & 4.7 & 1.5 & 2.0 & 8.0 \\
Semestre 10 & 33 & 6.4 & 1.8 & 3.0 & 9.0 \\
Semestre 11 & 33 & 6.9 & 2.0 & 2.0 & 10.0 \\
Semestre 12 & 33 & 7.4 & 1.6 & 4.0 & 10.0
\end{tabular}

correcta. Todos los estudiantes respondieron el mismo examen.

También registramos el número de casos de vida real que los estudiantes vivieron durante su rotación clínica, el número de participaciones en escenarios simulados y sus notas.

\section{Análisis de datos}

Para analizar las calificaciones de los estudiantes se usó el análisis de medidas repetidas de variación (ANOVA). Para comparar diferencias de calificaciones del examen entre los estudiantes que se enfrentaron a pacientes clínicos en la realidad y aquéllos que experimentaron con los pacientes clínicos en un ambiente de simulación, se aplicó una prueba t de muestras independientes. Para investigar la relación entre las calificaciones de los estudiantes en la prueba de conocimiento y número de atención de pacientes en la práctica real, se usó la correlación de Pearson (r). Todos los análisis fueron efectuados en software IBM SPSS Statistics 20 statistical. Para todas las pruebas estadísticas fue utilizado un nivel de significancia de $5 \%$.

\section{Ética}

Esta investigación ha sido aprobada por el Ethical Research Committee of Federal University of São Paulo-UNIFESP (Comité de Investigación Ética de la Universidad Federal de São Paulo). Número de proceso: no. 1888/11. La confidencialidad y anonimato fueron garantizados a todos los participantes del estudio, quienes de manera voluntaria aceptaron participar y dieron su consentimiento por escrito, informado.

\section{RESULTADOS}

Descubrimos un efecto principal de tiempo $\mathrm{F}(3.96)=28.69,(\mathrm{p}<0.01)$ lo cual indicó que las notas de conocimiento de los estudiantes aumentaron con el tiempo de forma significante (Tabla 1). Las notas de los estudiantes incrementaron del semestre 9 para el 10 y del semestre 11 para el 12 , pero no hubo diferencia significativa entre el semestre 10 y 11 .

Conforme a lo esperado, hay una tendencia creciente de participación en situaciones reales de paro cardiaco durante la práctica médica $(z=$ 
Tabla 2: Número de estudiantes, media, prueba t- de muestra relacionada y valores de relevancia de notas de los estudiantes en ambiente simulado y del hospital en los semestres $9,10,11$ y 12 .

\begin{tabular}{llrccc} 
& & & \multicolumn{3}{c}{ Desviación } \\
& & $\mathrm{n}$ & Media & típica & $\mathrm{T}$ \\
\hline \multirow{2}{*}{ Semestre 9 } & Simulado & 30 & 4.4 & 1.3 & -1.56 \\
& Hospital & 9 & 5.2 & 1.6 & \\
\multirow{2}{*}{ Semestre 10 } & Simulado & 21 & 6.0 & 1.7 & 0.39 \\
& Hospital & 19 & 6.3 & 1.8 & \\
Semestre 11 & Simulado & 17 & 6.3 & 1.9 & -1.12 \\
& Hospital & 26 & 7.0 & 1.9 & \\
Semestre 12 & Simulado & 4 & 6.0 & 1.2 & -1.47 \\
& Hospital & 41 & 7.2 & 1.6 & \\
\hline \multirow{2}{*}{ * $\mathrm{p}>0.05}$. & & & & &
\end{tabular}

$-6.54, \mathrm{p}<0.001)$, de $22 \%$ para $91.1 \%$ entre las etapas 9 y 12 . No encontramos relación entre el número de casos cardiacos reales tratados y las notas de los estudiantes $(r=0.145, p=0.344)$. Además, no hallamos diferencias entre los resultados medios entre los estudiantes que tratan pacientes en el ambiente real y aquéllos que tratan pacientes en el ambiente simulado (Tabla 2).

\section{DISCUSIÓN}

En este estudio investigamos la influencia del entrenamiento en simulación sobre la adquisición de conocimiento en estudiantes de medicina. Nuestros resultados mostraron que no hay relación ni diferencia entre los estudiantes que trataron un paciente con paro cardiaco en el ambiente clínico real comparado con quienes sólo trataron pacientes en el ambiente simulado, en su calificación del examen de conocimientos. Aunque la experiencia clínica en la vida real de los estudiantes nunca podrá ser sustituida, los estudiantes sin experiencia clínica directa son capaces de conseguir y conservar niveles similares de conocimiento a través de entrenamiento de simulación. Nuestros resultados han mostrado que el conocimiento de los estudiantes aumentó con el tiempo hasta el final de la práctica, lo que significa que el efecto del entrenamiento en simulación favorece el incremento y retención del conocimiento de los estudiantes.
Nuestros resultados son acordes a los de la literatura médica mundial que declara que el entrenamiento en simulación mejora la retención del conocimiento de los estudiantes. ${ }^{12,13}$ Además, el incluir un entrenamiento en simulación previo al inicio del entrenamiento clínico de los estudiantes parece mejorar su conocimiento, esto al ser comparado con simulación únicamente durante su entrenamiento clínico. ${ }^{14}$

La situación actual de las facultades de medicina en Brasil también debe ser considerada. En muchas de estas escuelas la práctica está basada sólo en la rotación por las principales áreas médicas, que en realidad conducen a la falta de experiencia clínica. Por ejemplo, en nuestro estudio tuvimos estudiantes que completaron toda la práctica y nunca vivieron una situación tal como un paro cardiaco en la vida real. La participación de esos estudiantes durante un paro cardiorrespiratorio simulado les ayudó a adquirir conocimiento y experimentar el curso de tratamiento en un ambiente más realista que, por ejemplo, basado en una conferencia. La posibilidad de repetir esta situación en estos dos años en un ambiente simulado contribuyó a aumentar la retención de conocimientos de los estudiantes. Asimismo, distanciar la sesión de entrenamiento con el paso del tiempo beneficia la retención del conocimiento y habilidad de los estudiantes. ${ }^{15,16}$

Aunque es posible conducir simulaciones de alta calidad usando grupos grandes, algunos educadores creen que no es posible efectuar simulaciones efectivas con grupos de muchos estudiantes. ${ }^{17,18}$ Los grupos pequeños posibilitan interacción estudiante-profesorado y colega-colega, mejorando las habilidades de interacción y proveyendo oportunidades para aclarar puntos de confusión. Por otra parte, los grupos reducidos ofrecen más demostraciones interactivas y participación del estudiante. ${ }^{19}$

Debido a la falta de un control grupal, no fuimos capaces de comparar estudiantes que practicaran en un escenario simulado con los que sólo asistieran a la conferencia. Aunque la falta de comparación obstaculiza el mejor entendimiento, nuestros datos soportan la inclusión de entrenamiento de simulación en la capacitación de estudiantes de medicina al demostrar que el entrenamiento en simulación ayuda a los estudiantes a adquirir y retener conocimientos. Además, no hubo incremento en el conocimiento de 
los estudiantes en los últimos tres momentos de medición. No obstante, eso puede ser explicado por el hecho que los estudiantes alcanzan una nota casi perfecta en la segunda prueba, conocida como el efecto techo, lo cual indica que el conocimiento de los estudiantes no ha decaído. Eso también es explicado por el hecho de que los estudiantes aún están en contacto con ese tipo de entrenamiento, por ejemplo, durante su rotación clínica ellos discutieron casos, incluyendo resucitación cardiopulmonar.

De forma distinta a nuestra muestra, un estudio reciente indicó que el conocimiento de profesionales de asistencia médica primaria decae en resucitación cardiopulmonar después de un año. ${ }^{20}$ Aunque tuvimos una muestra pequeña, logramos encontrar una diferencia significativa en el tiempo. Mientras el entrenamiento de simulación ha sido estudiado de manera amplia en ambientes de postgrado, no existe el uso de entrenamiento de simulación en programas de pregrado. ${ }^{9,10,21,22}$ Es necesaria una futura investigación para indagar si la inclusión de este entrenamiento es mejor que otro tipo de entrenamiento, por ejemplo, la conferencia o los grupos basados en problema.

\section{CONCLUSIÓN}

Los estudiantes que recibieron entrenamiento en simulación se desempeñaron de igual forma en una prueba de conocimiento que los que recibieron entrenamiento clínico con pacientes reales. Este hallazgo sugiere que la capacitación en simulación puede beneficiar la adquisición y retención de conocimientos de los estudiantes, así como la atención de pacientes reales. Sin embargo, se necesita una mayor investigación para aclarar si existe una diferencia entre la habilidades adquiridas por los estudiantes con ambos tipos de entrenamientos. Podría ser interesante realizar una investigación futura con un diseño de no inferioridad para dar seguimiento a nuestros hallazgos. Esto es importante, de manera particular, en áreas donde hay pocas oportunidades para que los estudiantes experimenten casos clínicos en vivo.

\section{FINANCIAMIENTO}

El estudio contó con el apoyo parcial de CAPES-33009015. El Dr. Marin reconoce el apoyo parcial de CNPq 303882/2013-1; 446221/20147 y el Subsidio 5D43TW007015-08 por el Fogarty International Center, la National Library of Medicine y los National Institutes of Health.

\section{REFERENCIAS}

1. Gaba DM. The future vision of simulation in health care. Qual Saf Health Care. 2004; 13 Suppl 1: i2-i10.

2. Steadman RH, Burden AR, Huang YM, Gaba DM, Cooper JB. Practice improvements based on participation in simulation for the maintenance of certification in anesthesiology program. Anesthesiology. 2015; 122 (5): 1154-1169.

3. Wolff M, Wagner MJ, Poznanski S, Schiller J, Santen S. Not another boring lecture: Engaging learners with active learning techniques. J Emerg Med. 2015; 48 (1): 85-93.

4. Gaba DM. Do as we say, not as you do: Using simulation to investigate clinical behavior in action. Simul Healthc. 2009; 4 (2): 67-69.

5. Issenberg SB, McGaghie WC, Hart IR, et al. Simulation technology for health care professional skills training and assessment. JAMA. 1999; 282 (9): 861-866.

6. Fuszard B. Innovative teaching strategies in nursing. Gaithersburg, MD: Aspen Publishers; 1995.

7. Scherer YK, Bruce SA, Graves BT, Erdley WS. Acute care nurse practitioner education enhancing performance through the use of clinical simulation. AACN A Adv Crit Care. 2003; 14 (3): 331-341.

8. Spunt D, Shpritz D. Clinical laboratory course development and management. In: Jairath N, Mills ME. Online health science education. Philadelphia: Lippincott Williams e Wilkins; 2006.

9. Aebersold M, Tschannen D, Bathish M. Innovative simulation strategies in education. Nurs Res Pract. 2012; 2012: 1-7. doi: 10.1155/2012/765212.

10. Coolen EH, Draaisma JM, Hogeveen M, Antonius TA, Lommen CM, Loeffen JL. Effectiveness of high fidelity video-assisted real-time simulation: a comparison of three training methods for acute pediatric emergencies. Int J Pediatr. 2012; 2012: 1-8.

11. Smith CM, Perkins GD, Bullock I, Bion JF. Undergraduate training in the care of the acutely ill patient: a literature review. Intensive Care Med. 2007; 33 (5): 901-907.

12. Cook DA, Hatala R, Brydges R, et al. Technologyenhanced simulation for health professions education: A systematic review and meta-analysis. JAMA. 2011; 306 (9): 978-988.

13. Boet $S$, Borges B, Naik V, et al. Complex procedural skills are retained for a minimum of $1 \mathrm{yr}$ after a single high-fidelity simulation training session. $\mathrm{Br} J$ Anaesth. 2011; 107 (4): 533-539.

14. Cecilio-Fernandes D, Brandão CFS, de Oliveira DLC, Fernandes GCVR, Tio RA. Additional simulation training: does it affect students' knowledge acquisition and retention? BMJ Simul Technol Enhanc Learn. 2019; 5: $140-143$.

15. Yeh DD, Park YS. Improving learning efficiency of factual knowledge in medical education. J Surg Educ. 2015; 72 (5): 882-889.

16. Cecilio-Fernandes D, Cnossen F, Jaarsma DA, Tio RA. Avoiding surgical skill decay: a systematic review on 
the spacing of training sessions. J Surg Educ. 2018; 75 (2): 471-480.

17. Fitch MT. Using high-fidelity emergency simulation with large groups of preclinical medical students in a basic science course. Med Teach. 2007; 29 (2-3): 261-263.

18. Heitz C, Brown A, Johnson JE, Fitch MT. Large group high-fidelity simulation enhances medical student learning. Med Teach. 2009; 31 (5): e206-e210.

19. Euliano TY. Small group teaching: clinical correlation with a human patient simulator. Adv Physiol Educ. 2001; 25 (1): 36-43.

20. Nogueira LS, Wilson AMMM, Karakhanian ACM, Parreira EV, Machado VMP, Mira VL. Avaliação dos conhecimentos e habilidades em ressuscitação cardiopulmonar assimilados por profissionais da atenção primária em saúde. Sci Med. 2018; 28 (1): ID28843. http://doi.org/10.15448/1980-6108.2018.1.28843.
21. Brandão CFS, de Carvalho-Filho MA, CecilioFernandes D. Simulation centers and pedagogical planning: two sides of the same coin. Sci Med. 2018; 28: ID28709. http://doi.org/10.15448/19806108.2018.1.28709.

22. Brandão CFS, Fernandes DC. Importance and challenges of simulation training in healthcare. Sci Med. 2018; 28: ID30102. http://doi.org/10.15448/19806108.2018.1.30102.

Correspondencia:

Carolina Felipe Soares-Brandão

UNICID, Rua Butantã, 285, Pinheiros.

São Paulo-SP.

E-mail: carolinafs11@gmail.com 